\title{
Parkinson's Disease and Gene Therapy
}

\author{
Mustafa M. Amin ${ }^{1 *}$ D, Aldy S. Rambe ${ }^{2}$, Kiking Ritarwan² \\ ${ }^{1}$ Department of Psychiatry, Faculty of Medicine, Universitas Sumatera Utara, Medan, Indonesia; ${ }^{2}$ Department of Neurology, \\ Faculty of Medicine, Universitas Sumatera Utara, Medan, Indonesia
}

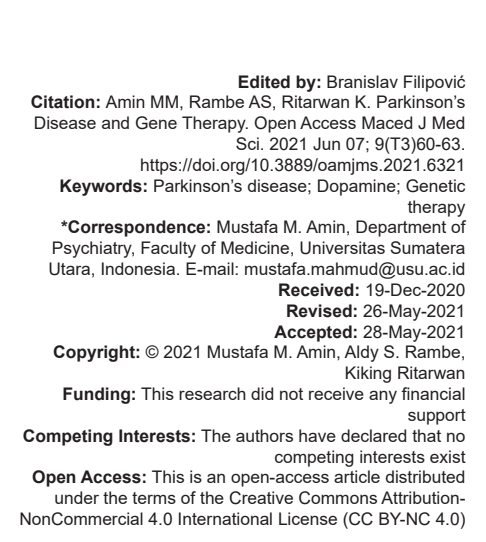

\section{Abstract}

Since its introduction for more than a century, Parkinson's disease has caught attention in the field of neuroscience. From the beginning of understanding its pathogenesis of dopamine pathways, many studies have grown in finding the treatment of the disease. In the beginning, medications showed promise results until each of them had its limitation in treating the disease. Another focus of attention from studies in the last decade was from gene therapy, and early phases of studies have shown some pledging moments for the disease.

\section{Introduction}

It started at the beginning of the ninetieth century when James Parkinson identified a disorder in his patients who had tremor resting with less muscle strength and flexed posture of the body, though intellectuality and sensitivity remained intact [1], [2]. $\mathrm{He}$ did not mention about reduced movement and rigidity [1]. Patients with Parkinson's disease (PD) have now been characterized by postural reflex deterioration, bradykinesia (lack of spontaneous motion and slowing of motion, mostly repetitive motion), tremor resting (particularly when patients close their eyes, limbs, and jaw), rigidity (such as cogwheel appearance) [1], [2], [3], and experience non-motor symptoms, for example, constipation, sleep disorders, pain, depression, and genitourinary problems [4].

Parkinsonism can be seen as a side effect of antipsychotic treatment; however, unlike PD, when the patient no longer takes the treatment, antipsychoticinduced Parkinsonism disappears [5]. In PD, patients have been suffering from the disorder since the start of their diagnosis. In patients, the progression of the disease can be seen as early as 40 years or as late as 60 years. It has become one of neurology's most prevalent diseases, and it affects the population, particularly older people [1], [2], [3]. PD treatment only focuses on the medications themselves in the early days. Today, the treatment has ranged from medications to gene therapy to surgery [1], [2], [3], [6], [7].

\section{Definition}

A current concept of PD, a progressive neurodegenerative disorder that results from the loss of dopaminergic neurons in substantia nigra, is given by the National Collaborating Centre for Chronic Conditions (2006. p. 3). History and clinical review make up the diagnosis, and no test may differentiate PD from other diseases of the same clinical appearance [8].

\section{PD Pathogenesis}

In the brain, there are few dopamine pathways, Stahl, 2008 and Fernandez-Espejo have reviewed that the nigrostriatal pathway is one pathway that relates to PD. From substantia nigra to striatum, it projects. Usually, the pathway has many dopaminergic neurons; however, studies have shown that the loss of 
dopaminergic neurons in the PD nigrostriatal pathway, especially dopaminergic neurons located in substantia nigra pars compacta (SNpc), has occurred [5], [6].

\section{Oxidative destruction, as the result of}

$\bullet$

Rise of hydrogen peroxide, dopamine-quinone species, superoxide radicals from dopamine metabolism. In iron catalysis, the Fenton reaction produces new cytotoxic hydroxyl radicals from hydrogen peroxide. Efficient oxidizing agents derived from superoxide reactions with high levels of nitric oxide.

- Increase of increase in levels of nigral iron to a low level of glutathione (GSH). More exposure to toxin action and possible activation of the toxic effects of glial cells have been correlated with low GSH levels.

- $\quad$ The enormous growth of nitrogen and reactive oxygen species, which increases protein, lipid, and deoxyribonucleic acid oxidative harm.

All of the above lead to dopaminergic cell death in substantia nigra.

\section{Noxious toxic sequence}

As a result of the reduction in the activity of complex I, mitochondrial dysfunction. It occurs when iron-mediated free radical development limits the activity of complex I, and whenever in the toxic chain, mitochondrial inhibition and oxidative stress strengthen each other.

- $\quad$ Excitotoxic damage as a consequence of forceful substantia nigra degeneration, which causes subthalamic nucleus hyperactivity. Substantia nigra releases exaggerated glutamatergic neurons inside it following this occurrence. Dopamine destruction in substantia nigra is caused by excessive release of glutamate.

- The neuroinflammatory reaction. This is the result of a notable rise in PD patients' levels of cytokines in the cerebrospinal fluid and striatum. As the neurodegeneration phase begins, it stimulates T-lymphocytes in the substantia nigra, which begins the activation of glial cells. Glial cells that contribute to oxidative stress and apoptosis release proinflammatory factors [6].

Another finding is the presence of Lewy Bodies (LBs) in the brain of the patients [1], [2], [9]. LBs are intraneuronal proteinaceous cytoplasmic inclusions [9]. It is located in neurons that are pigmented. The primary projections of these neurons are putamen and caudate [2]. The four proteins that build LB are neurofilaments, ubiquitin, parkin, and $\alpha$-synuclein [9]. The characteristic of LB are:

\begin{abstract}
1. The diameter is $15 \mu \mathrm{m}$.
2. It has a dense hyaline-organized structure.

3. A halo surrounds the structure [1], [2], [9].

Except for inherited PD (Ropper and Samuels, 2009), many patients with PD have been found with LB in their brain; however, LB itself is also found in dementia. The exact role of LB in the disease remains unclear up to now [1], [9].
\end{abstract}

\section{Animal Model of PD}

It started in 1982, and four young substance addicts in Northern California developed signs of PD. Because of the severity of the disorder, it was exceptional when young people developed PD.

A new type of designer drug was decided by neurologists [9], [10], [11]. It worked similar like heroin and contained 1-methyl-4-phenyl-proprionoxy-piperidine (MPPP), which correlated with narcotic meperidine, and a contaminant of MPPP, which was 1-methyl-4-phenyl1,2,5,6-tetrahydropiridine (MPTP). They discovered that the one which related to the $\mathrm{PD}$ symptoms is MPTP. Monoamine oxidase oxidized MPTP in the brain to MPP+. Later on, dopamine neurons took MPP+ and inhibited oxidative metabolism in mitochondria, leading to neuronal death [10]. Idiopathic PD symptoms were similar to the MPTP-induced Parkinsonism, pathologically [11]. There are three theories regarding the MPTP-induced Parkinsonism pathways. First, the decline in the subthalamic nucleus of gammaaminobutyric acid results in the globus pallidus, part of the inner segment, becoming hyperactive.

Second, several MPTP-related environmental factors cause Parkinsonism. Third, the toxin itself was predisposed to inducing PD by the gene in humans [1]. All of these findings lead to the studies of MPTP in primate [9], [10], and mice [9].

In studies of monkeys, bradykinesia, lack of movement, rigidity, and tremor have been shown to cause MPTP-parkinsonism. It is associated almost entirely with degeneration of dopamine neurons in the SNpc and ventral tegmental region and inconsistent degeneration in the locus coeruleus. When L-DOPA is administered to them, the monkeys undergo Chorea. In humans, it mimics the unwanted effects of L-DOPA [1], [9], [10]. Dauer and Przedborski in 2003 reviewed, previous authors have found in the mice studies that: (1) MPTP-induced degeneration resistance of dopaminergic neurons occurred in superoxide dismutase-1 transgenic mice and (2) apoptosis occurs in the morphological form of SNpc dopaminergic neurons of mice when injected with tolerable doses of MPTP. These results have led to a decrease in the number of MPTP-induced dopaminergic neurons [9]. 


\section{PD Gene Therapy}

Gene therapy, which raises the number of dopaminergic neurons in the putamen and caudate by introducing modified genetic cells to alter tyrosine to become dopamine, is a relatively novel treatment for $P D$ [12]. Comparing to any neurological disease, $P D$ is the one which has the most gene therapeutic approaches [13].

The two types of gene therapy in PD are:

\section{In vivo}

The technique is to pass the gene to the basal ganglia dysfunction to apply dopaminergic neuron trophic factors, boost dopaminergic production insufficiency, balance negative intracellular pathways, and alter neurotransmitters and electrophysiological imbalance sequencing [13]. Horellou and Mallet in 1997 reviewed three in vivo vector candidates: Adenoassociated virus (AAV), adenovirus, and herpes simplex virus-1, all of which interceded with intracerebral gene expression after injection [14]. Using adenovirus as a vector, there is also glial-derived neurotrophic factors (GDNF) gene transfer. In a review by Ralph et al. in 2006, they have written, GDNF is potent to support dopamine neuron from degeneration in PD [15].

\section{Ex vivo}

The technique is the approach is to transfer a gene to produce primary cells or cell lines that have been genetically engineered in the brain of PD patients for transplantation. This technique was intended to increase the activity and continuation of cells, to obtain the phenotype of dopamine by switching cells and accommodating the nigrostriatal terminal trophic factors [13]. Exceptional use of conditional oncogenes for cell transformation was provided. Intracerebral transplantation can use primary human cells that have been activated by mitogens to develop [14].

Viral vectors are the most widely used for gene therapy in PD. Witt and Marks, Jr (2011) have concluded that the ideal viral vectors have to have: Safety, the capability to transfect the neuronal propensity host cells adequately, the capability of gene therapy long-term expression, and in some cases, have unique properties [7].

Nowadays, studies have suggested that the two favored vectors for gene therapy of PD are AAV and lentivirus (LV), and there are few essential gene therapy arrangements for PD patients shortly [16]. They are:

\section{Neurotrophic factors}

\section{Glial-derived neurotrophic factors}

Ralph et al. (2006) have written that studies using LV vectors generated to express GDNF in rodents and primate models of PD have shown promising results [15]. GDNF has a neuroprotective effect and efficiency in transduction at the neurons of dopamine in the nigrostriatal pathway and prevents dopamine neuronal loss at the striatum and substantia nigra, which refine motor functioning of the research animals, and Berry and Foltynie (2011) have reviewed that in MPTP and 6-hydroxydopamine (6-OHDA) models of animals, GDNF has been acknowledged to prevent and lessen the death of dopamine in the nigrostriatal pathway, and diminishing the behavioral symptoms of Parkinsonism [16]. Unfortunately, the results are not as good as in double-blind, randomized controlled trials for humans.

\section{AAV neurturin}

Berry and Foltynie (2011) have reviewed that using AAV2 vectors, the MPTP model of primates and 6-OHDA model of rodents have shown the efficiency of neurturin. It is injected toward the striatum and substantia nigra of the primates and in the striatum of the rodents. It has tested in several patients and shown improvements of the Unified PD Rating Scale (UPDRS) III scores after 12 months. Contradictory data have been shown in the results of a double-blind, randomized trial of neurturin. There have been no changes between baseline and endpoint in the UPDRS III of 58 patients [16]. In another review by Witt and Marks, Jr (2011), significant improvements in UPDRS OFF scores have been shown in phase I, open-label study using neurturin; however, the results have not been followed by the brain PET scan of the patients [7].

\section{Dopamine increasing gene therapy} $(h A A D C)$

AAV aromatic-L-amino decarboxylase

Berry and Foltynie (2011), and Witt and Marks, Jr (2011) have reviewed, in a 6 months phase I study with ten patients, and there has been significant improvement of the UPDRS III ON-OFF scores after the infusion of AAV2 hAADC at the bilateral posterior putaminal of the brain [7], [16].

\section{LV tyrosine hydroxylase (TH) AADC GTP} cyclohydrolase-prosavin (GCH1-prosavin)

Berry and Foltynie (2011) have reviewed, in a 24 months phase I and II study of clinical trials of patients with PD using prosavin, there have been positive results in the UPDRS III OFF scores compared to baseline [16].

\section{Circuitry intervening gene therapy}

AAV glutamic acid decarboxylase (GAD)subthalamic nucleus (STN) inhibition

As Berry and Foltynie (2011) and Witt and Marks, Jr (2011) have reviewed, following significant 
healing of behavioral activity in 6-OHDA model of rat, and the positive results of MPTP model of primate, using AAV2 vector encoding GAD injected toward STN, a phase I study which focusing unilateral STN using AAV2 vectors in 12 PD patients have also shown positive results. After 12 months, there have been improvements in the UPDRS III ON-OFF scores [7], [16].

\section{Conclusion}

Parkinson's disease is a neurological condition that is widespread. It can be observed as individuals grow older; it has also been recognized as a neurodegenerative disease. People with PD encounter motor and non-motor symptoms, that are primarily the product of a deficiency of dopaminergic neurons, especially in the brain of the patient's SNpc. The presence of LB in the brain is another cause of PD, but LB itself is also present in another disorder.

There is not a single medication available so far to cure PD. Studies have shown positive results in gene therapy recently. Gene therapy attempts to increase the level of dopamine in the brain of the patient so that the effects of PD can be relieved. Before PD patients enjoy it for themselves, few gene therapies are still in ongoing clinical phases. Following the promising outcomes of the clinical phase trials, it is highly recommended that alternative gene therapies be identified for the potential benefit of PD patients.

\section{References}

1. Ropper AH, Samuels MA. Adams and Victors Principles of Neurology. $9^{\text {th }}$ ed. New York: McGraw-Hill; 2009.

2. Apetauerova D. Parkinson's disease. In: Jones HR Jr., Srinivasan J, Allam GJ, Baker RA, editors. Netter's Neurology. $2^{\text {nd }}$ ed., Ch. 33. Philadelphia, PA: Elsevier, Saunders; 2012.

3. Wilkinson I, Lennox G. Essential Neurology. $4^{\text {th }}$ ed. Massachusetts: Blackwell Publishing, Inc.; 2005.

4. Chauduri KR, Healy DG, Schapira AH. Non-motor symptoms of Parkinson's disease: Diagnosis and management. Lancet Neurol. 2006;5(3):235-45 PMid:16488379

5. Stahl SM. Stahl's Essential Psychopharmacology Neuroscientific Basis and Clinical Applications. $3^{\text {rd }}$ ed. New York: Cambridge University Press; 2008.

6. Fernandez-Espejo E. Pathogenesis of Parkinson's disease. Mo Neurobiol. 2004;29(1):15-30.

7. Witt J, Marks WJ Jr. An Update on gene therapy in Parkinson's disease. Curr Neurol Neurosci Rep. 2011;11(4):362-70. https:// doi.org/10.1007/s11910-011-0197-8

PMid:21479996

8. The National Collaborating Centre for Chronic Conditions. Parkinson's Disease. National Clinical Guideline for Diagnosis and Management in Primary and Secondary Care. London: Royal College of Physicians; 2006.

9. Dauer W, Przedborski S. Parkinson's disease: Mechanisms and models. Neuron. 2003;39(6):889-909. https://doi.org/10.1016/ s0896-6273(03)00568-3

PMid:12971891

10. Mink JW. The basal ganglia. In: Squire L, Berg D, Bloom F, du Lac S, Ghosh A, Spitzer NC, editors. Fundamental Neuroscience. $3^{\text {rd }}$ ed., Ch. 31. Canada: Academic Press; 2008.

11. Fuller G, Manford M. Neurology an Illustrated Colour Text. $3^{\text {rd }}$ ed. Edinburgh: Churchill Livingstone; 2010.

12. Purves D, Augustine GJ, Fitzpatrick D, Hall WC, LaMantia AS, McNamara JO, et al., editors. Modulation of movement by the basal ganglia. In: Neuroscience. $5^{\text {th }}$ ed., Ch. 18. Sunderland: Sinauer Associates, Inc.; 2012. https://doi.org/10.1002/ (sici)1521-1878(199810)20:10<871::aid-bies15>3.0.co;2-f

13. Eberhardt $O$, Schulz JB. Gene therapy in Parkinson's disease. Cell Tissue Res. 2004;318:243-60.

14. Horrelou P, Mallet J. Gene therapy for Parkinson's disease. Mo Neurobiol. 1997;15(2):241-56.

15. Ralph GS, Binley K, Wong LF, Azzouz M, Mazarakis ND. Gene therapy for neurodegenerative and ocular diseases using lentiviral vectors. Clin Sci. 2006;110:37-46. https://doi. org/10.1042/cs20050158

PMid:16336203

16. Berry AL, Foltynie T. Gene therapy: A viable therapeutic strategy for Parkinson's disease? J Neurol. 2011;258(2):179-88. https:// doi.org/10.1007/s00415-010-5796-9

PMid:20963433 